\title{
Structured Coding for Content Based Interactive Audio
}

\author{
Kathy Melih and Ruben Gonzalez \\ School of Information Technology, Griffith University \\ PMB 50 Gold Coast Mail Centre, QLD 9726, Australia. \\ \{K.Melih,R.Gonzalez\}@gu.edu.au
}

\begin{abstract}
This paper addresses the dual problem of audio coding for compression and retrieval. Because of the sheer volume of multimedia data, much research effort has focused on coding techniques for the compression of this data. However, being able to store large amounts of data in a compact fashion has limited application if there is no meaningful way to access and interact with them. Traditionally, these two issues have been treated separately resulting in many problems for multimedia data management. The authors have proposed a solution to these problems for audio by developing a compact, perceptually based, structured audio representation that provides explicit support for content based retrieval, browsing and other interactions. In addition, the representation offers potential for compressed data storage. This paper outlines a coding scheme based on the representation and reports the compression results.
\end{abstract}

\section{Introduction}

Effective management of multimedia data is an increasing concern. The most obvious issue requiring attention is the voluminous nature of multimedia data. Accordingly, much research effort in the past has focussed on devising coding techniques that allow data compression. However, compression is not the only problem faced in multimedia data management. Having compressed the data to a physically manageable size often results in a volume of information that is difficult to manage conceptually. For example, 10 mins of $\mathrm{CD}$ quality raw audio data requires approximately $100 \mathrm{Mbytes}$ of storage. Using MPEG layer 3 compression it is possible to store at least one hour of similarly high quality audio in the same amount of storage space. Obviously, the task of recovering a specific short section of audio is much more difficult in the latter case. Thus methods to provide meaningful access to and content based retrieval of compressed multimedia data are required.

Traditionally, the problems of data compression and information management support have been treated in isolation from one another. This has resulted in a number of problems. In particular, conventional coding techniques designed purely for compression have resulted in statistically based representations that obscure the inherent structure and content of the underlying data. In contrast, this paper presents a coding scheme based on a perceptually tuned structured representation.

On the other hand, the few existing systems focussing purely on content-based retrieval have generally relied on separate index files, thus increasing storage requirements. These indexes are often based on statistical attributes that are too low level to have cognitive significance. Audio data poses additional conceptual problems, such as how to deal with multiple sources (eg, speech in musical background). Perhaps as a result of these difficulties audio data has received the least attention in multimedia data management research.

In an attempt to address audio retrieval and compaction issues simultaneously, a perceptually based, structured audio coding model has been developed[1]. The advantage of this model is, not only has it been designed specifically to support content based retrieval but it also provides for compact representation. With such a representation audio data management can become very efficient both in terms of storage requirements and retrieval/access support.

\section{Background}

Traditional audio coding techniques focus on statistically based compression[2]. This is because the main aim of coding has been data rate reduction. However, the increasing need to provide meaningful interaction modes for multimedia data means that this is no longer the sole concern. Nevertheless, compression is still an important requirement hence, providing meaningful access should not compromise compression. In particular, providing such interaction should not require significantly more storage than the raw data itself.

The focus on compression has resulted in audio representations that are unstructured and obfuscate the salient perceptual and structural information present in the underlying data[3]. This in turn has caused many problems for the desire to support content-based audio retrieval. Generally, the solution of these problems has involved generating separate index or meta-files. An example of these approaches is the annotation based indexing described in the current MPEG 7 proposal. There are, however, many disadvantages to such an approach. 
At the very least, the need to generate separate metafiles results in processing and storage overheads. Obviously, this addition is counter to the desire for compression. Further problems arise from the methods currently employed to generate these meta-files. The two existing methods for generating index files are: sematic and statistical.

In the case of semantically based techniques, index generation is highly constrained. The main aim of semantic techniques is to generate a textual description of the audio data's content. This is a problem in itself since audio signals possess many qualities that are difficult to describe in such a manner. Examples include prosodics and timbre. Further, the data and queries must generally be collected under strictly controlled conditions and often, manual intervention is required for accurate transcription. One such example is generating a textual transcription for speech.

Basing indexes on statistical attributes (such as in [4]) removes some of these constraints but results in descriptions that have very little cognitive significance. For example, search keys such as harmonicity and bandwidth, frequently used by statistically based systems, are of little relevance to the general user. A method that exploits the structure of the underlying audio data, as revealed in a perceptually based representation provides some solutions to these problems.

A number of key attributes appear to be extracted from an audio signal during the process of human audio perception. Basing a representation on these attributes is an obvious means of aiding content-based retrieval since a low-level index is effectively 'built in' to the representation. As these attributes are perceptually based, they will most likely support higher level queries. Also, such a representation readily lends itself to compression as perceptually redundant information is eliminated from the data. Indeed, high quality, low rate coding often exploits the properties of human audio perception [5].

A perceptually based audio representation appears to solve many of the problems associated with information management. However, these benefits can only exist if the representation is coded carefully so as not to obscure the perceptually significant structure of the underlying data. With careful encoding, a perceptually based representation can address the issues of meaningful access and data rate reduction simultaneously.

\section{Representation summary}

The representation developed was described in detail in [1]. A summary and update of its essential features is given here. The basic process used to obtain the representation is described in Figure 1. Incoming audio data is analysed to produce a TFD. The peaks in amplitude of this distribution are found and tracked through time and frequency. Each resultant track is then classified according to type (tone, noise or sweep) and parametrically recorded. Finally, the tracks are grouped according to psychoacoustic principles and encoded in these groups.

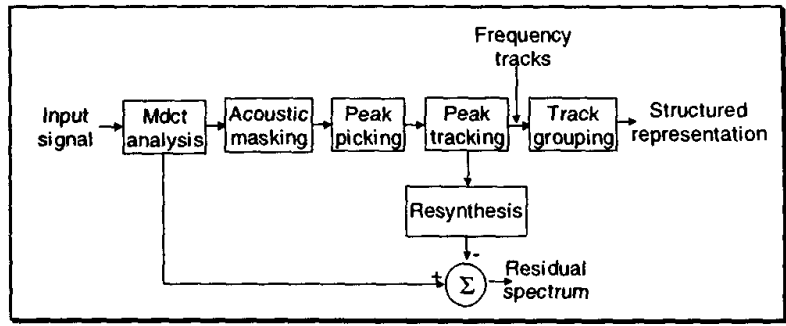

Figure 1: Coding algorithm

The representation is essentially an extension of sinusoidal coding. The main differences are a perceptually tuned time-frequency resolution, use of a real transform in place of the FFT in TFD generation and the grouping of tracks based on perceptual principles. The time-frequency resolution is shown in Figure 2.

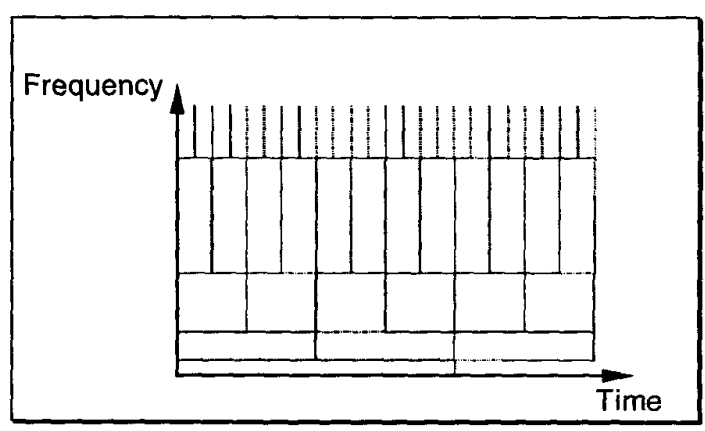

Figure 2: Time-frequency resolution of the TFD

To eliminate redundancy, a real transform is desired. Originally a modified discrete cosine transform (MDCT) was used for this purpose however it was found that the implicit coding of phase by the MDCT caused problems for both peak tracking and reconstruction quality.

To overcome these problems, a discrete Hartley transform has been used instead[6]:

$X(k)=\sum_{n=0}^{N-1} x(n)\left(\cos \left(\frac{2 \pi n k}{N}\right)+\sin \left(\frac{2 \pi n k}{N}\right)\right), k=0,1, \ldots N-1$

The advantage of the Hartley transform is that, while it is purely real, it is possible to explicitly derive phase information from it. This has been found to be necessary for good quality reconstruction of tracks at lower frequencies.

It was reported previously [1] that the peak tracking algorithm originally used in the sinusoidal coding scheme tends to display an undesirable horizontal bias. This horizontal bias results from the fact that the original algorithm effectively employs a zeroth order predictor. An extension that would overcome the horizontal bias is 
to use a higher order linear predictor. The problem then arises as to how to estimate the initial predictor values. One option is to initially assume that each new track is horizontal, however this is not likely to alleviate the horizontal bias to a great extent. Another possibility is the use a Hough transform [7] over a short window of peaks extending from the beginning of the new track.

Once the tracks have been generated, each track is classified according to type: noise burst, tone burst or frequency sweep. Very short tracks are classified as noise bursts. Examining the frequency contour of the track makes the decision as to whether a long track belongs to the class of tone burst or sweep.

Next, psychoacoustic principles are applied to segregate them into streams. At this stage, the aim of segregation is simply to remove correlation in the data so only a very basic set of principles is applied. These principles are illustrated in Figure 3.

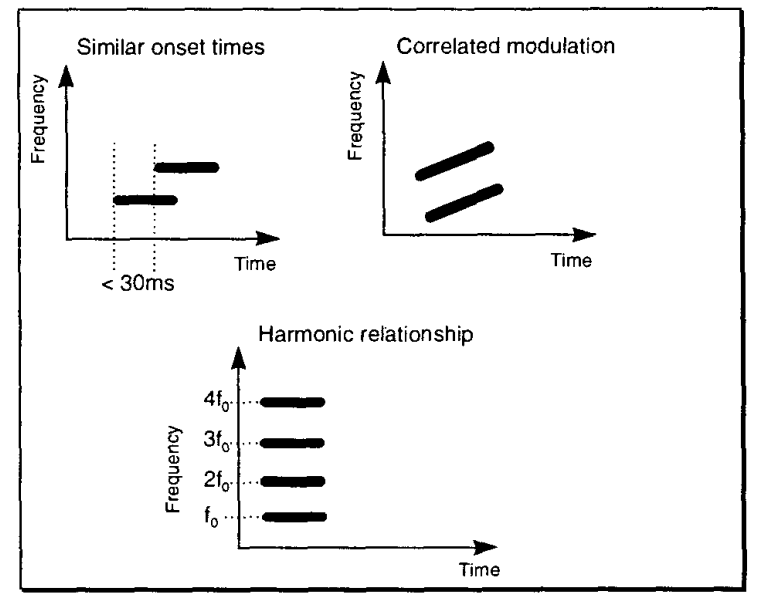

Figure 3: Psychoacoustic grouping rules

Having generated the representation, it may be inverted using the procedure illustrated in Figure 4.

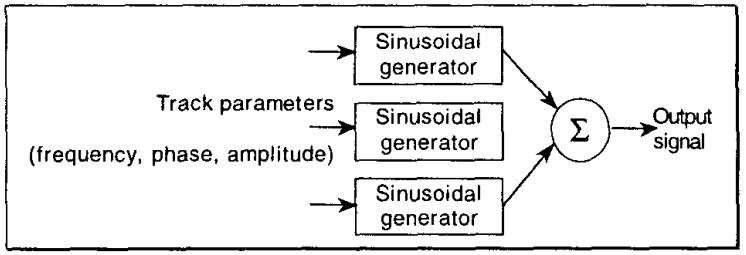

Figure 4: Track inversion

\section{Coding the representation 4.1. General considerations}

The representation is basically an extension of sinusoidal coding[8]. However, traditional sinusoidal codecs, following the lead of most audio coding, are designed purely for compression. As a result, the track structure is completely obscured in the coded data and in most cases is never even explicitly derived. Thus it has been necessary to develop a new coding scheme to accommodate the aims of the work described here.

During the development of this representation, the desire to provide direct access to the inherent structure of the audio data was paramount. This same consideration drives the encoding process. While a high compression ratio is desirable, it can not come at the expense of obfuscating the representation's structure. Thus, the ultimate aim of the coding process is to produce the most compact representation possible without obscuring the inherent structure of the data that it represents.

To achieve this aim, the coded data must display a number of characteristics. Specifically, the inherent structure of the underlying data needs to be directly apparent from its encoded form. Each element within this structure must be individually decodable, randomly accessible and indexable.

At present, the chief aim is to investigate the suitability of the representation for retrieval. Hence, the aim of coding has been modest: to produce a reasonably compact representation that can be used as the basis for experimentation. Developing optimum coding techniques is an area for further research. However, this section shows that the representation is well suited to compaction as well as supporting content based retrieval. This in direct contrast with existing multimedia data management and audio coding techniques that treat these two tasks as completely separate problems.

\subsection{Coding the tracks}

The tracks may be coded using a three dimensional chain code. This is an extension of the two dimensional Freeman chain code introduced in [9]. Chain code represents a trajectory in terms of relative transitions in a parameter space. Chain codes are an ideal way to code the tracks since the most significant information contained in the tracks is their contour. By using a three dimensional chain code, both frequency and amplitude contours can be coded at the same time further compacting the final representation. This may seem to come at the expense of a more complicated search since the frequency and amplitude contours are not explicitly separated as required in some retrieval tasks. However, judicious code assignment can separate these two contours implicitly.

To simplify chain coding, two assumptions are made concerning track nature. The first assumption is that successive codes along a chain correspond to a single step in time, where the size of this step is defined to be the length of the smallest analysis window. The second assumption is that the amplitude and frequency can only vary by one 'unit' (the size of which is determined by the previous value) from one frame to the next. The first assumption requires interpolating values in time for tracks that exist in the lower frequency bands which would have 
been required at synthesis time. The second assumption is valid since audio signals vary slowly in time.

Given these two assumptions and setting the current point on a particular track as the origin of a three dimensional space, the next point in the track may occupy one of nine positions:

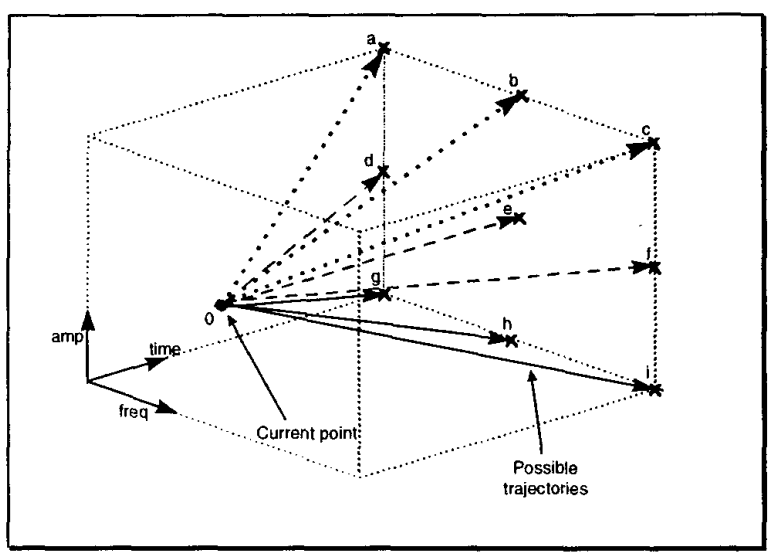

Figure 5: Allowed track trajectories for 3-D case

From Figure 5 it can be seen that nine codes are required requiring only four bits store each point. A track termination code is required which can be any one of the unused four bit combinations. A small amount of 'header' information is also required for each track: the position (in time) of the first point in the track, and the initial amplitude and frequency values. Further coding gains can be achieved with variable length chain codes.

\subsection{File structure}

In order for the basic aim of the representation to be achieved, it is imperative that the coded data be written to file in a manner that keeps the structure of the underlying data explicit. In addition, random access must be supported. These considerations ensure that the desire for compression does not override the fundamental aim of creating a representation that provides intrinsic support for audio retrieval tasks.

The proposed file structure consists of a header that is a list of track positions in the file. This list is terminated by a null code. Each track is preceded by it's own header information as described in section 5.3. Since each track is effectively self contained, it is a simple matter for some higher level process to assign a new organisation to the tracks by creating a list of pointers to individual tracks or groups of tracks.

\section{Preliminary results \\ 5.1. Test data}

All test data used were mono 16-bit, $32-\mathrm{kHz} \mathrm{PCM}$, WAV files. This file format was chosen as reasonably high audio quality was desired and $32-\mathrm{kHz}$ represents a compromise between quality and computational convenience (a $32-\mathrm{kHz}$ sampling rate accommodates an even number of sub-bands for the TFD analysis).

Two classes of test data were used. The first set contained two short 4.5-sec sections of female speech: the digits 1 to 4 and the vowels. The second set of test data consisted of two musical excerpts. Both excerpts were 4.5 seconds in length. The first was from a trumpet concerto by Telemann and the second from Bach's Brandenburg Concerto No. 5.

\subsection{Code assignment and entropy calculation}

This section defines the code assignment for each of three cases and reports the resultant entropy. The three cases are: 3-D chain code with fixed-length code words, 3-D chian code with variable-length code words and 2-D chain code with variable-length code words.

Table 1 shows the possible code assignment for 3-D fixed-length chain code. The symbol ' $x$ ' is the track terminator and is not counted as a symbol for the purposes of entropy calculation.

Table 1: Chain codes for points in a track

\begin{tabular}{|c|c|c|c|}
\hline & $\begin{array}{c}\text { Relative } \\
\text { Frequency } \\
\text { Change }\end{array}$ & $\begin{array}{c}\text { Relative } \\
\text { Amplitude } \\
\text { Change }\end{array}$ & Code \\
\hline \hline a & -1 & 1 & 1101 \\
\hline $\mathrm{b}$ & 0 & 1 & 0001 \\
\hline $\mathrm{c}$ & 1 & 1 & 0101 \\
\hline $\mathrm{d}$ & -1 & 0 & 1100 \\
\hline $\mathrm{e}$ & 0 & 0 & 0000 \\
\hline $\mathrm{f}$ & 1 & 0 & 0100 \\
\hline $\mathrm{g}$ & -1 & -1 & 1111 \\
\hline $\mathrm{h}$ & 0 & -1 & 0011 \\
\hline $\mathrm{i}$ & 1 & -1 & 0111 \\
\hline $\mathrm{x}$ & & & 1010 \\
\hline
\end{tabular}

Noting that the sum of probabilities of occurrence must be equal to one, the entropy of this case is given by:

$$
\begin{aligned}
H & =\sum_{i=1}^{N} P_{i} I_{i}, \text { but } I_{i} \text { constant so, } \\
H & =I \sum_{i=1}^{N} P_{i} \\
& =I
\end{aligned}
$$$$
=4 \text { bits in this case. }
$$

The relative probability of each code for 3-D standard chain code, as well as the codewords generated using a Huffman tree based on these probabilities is given in Table 2. 
Table 2: Relative frequency of occurrence of symbols for standard chain code

\begin{tabular}{|c|c|c|c|c|}
\hline Symbol & Music & Speech & Average & Code \\
\hline $\mathrm{a}$ & $5.97 \%$ & $6.36 \%$ & $6.16 \%$ & 11110 \\
\hline $\mathrm{b}$ & $20.20 \%$ & $13.54 \%$ & $16.87 \%$ & 100 \\
\hline $\mathrm{c}$ & $6.82 \%$ & $8.14 \%$ & $7.48 \%$ & 1101 \\
\hline $\mathrm{d}$ & $7.01 \%$ & $10.50 \%$ & $8.76 \%$ & 1011 \\
\hline $\mathrm{e}$ & $19.69 \%$ & $18.79 \%$ & $19.24 \%$ & 00 \\
\hline $\mathrm{f}$ & $7.05 \%$ & $11.20 \%$ & $9.13 \%$ & 1010 \\
\hline $\mathrm{g}$ & $7.16 \%$ & $8.20 \%$ & $7.68 \%$ & 1100 \\
\hline $\mathrm{h}$ & $21.07 \%$ & $15.59 \%$ & $18.33 \%$ & 01 \\
\hline $\mathrm{i}$ & $5.04 \%$ & $7.67 \%$ & $6.35 \%$ & 1110 \\
\hline $\mathrm{x}$ & & & & 1111 \\
\hline
\end{tabular}

The entropy for this case is thus:

$$
\begin{aligned}
H & =\sum_{i=1}^{N} P_{i} I_{i} \\
& =3.1 \mathrm{bits}
\end{aligned}
$$

The calculation in (7) shows that variable length code words offer significant saving over fixed length codes. However, this comes at a price: frequency and amplitude contours are no longer be explicitly coded. This can be overcome by coding the frequency and amplitude contours as separate tracks where each track has only three allowed directions: higher, lower and same. The probabilities of occurrence for the allowed directions along the amplitude and frequency contours are shown in Table 3.

Table 3: Relative frequency of occurrance for each direction when amplitude and frequency contours are coded separately

\begin{tabular}{|c|c|c|c|c|c|}
\hline & Direction & Music & Speech & Average & Code \\
\hline & up & $20.14 \%$ & $25.06 \%$ & $22.60 \%$ & 110 \\
\hline & same & $60.96 \%$ & $47.92 \%$ & $54.44 \%$ & 0 \\
\hline down & $18.91 \%$ & $27.02 \%$ & $22.96 \%$ & 10 \\
\hline & up & $32.99 \%$ & $28.03 \%$ & $30.51 \%$ & 110 \\
\hline & same & $33.75 \%$ & $40.50 \%$ & $37.12 \%$ & 0 \\
\hline down & $33.27 \%$ & $31.47 \%$ & $32.37 \%$ & 10 \\
\hline
\end{tabular}

Resulting in an entropy of:

$$
\begin{aligned}
H & =1.7 \text { bits for freq and } \\
& =1.9 \text { bits for amplitude }
\end{aligned}
$$

Thus to code both contours an average of 3.6 bits per frame would be required. This represents an improvement over 3-D chain code using fixed code lengths but is not as efficient as the variable length 3-D chain code. This is to be expected since, by combining the two variables in the 3-D code we exploit source extension.

\subsection{Compression ratios}

Several possibilities for coding the tracks have been discussed. The actual compression achieved using each of the following schemes will now be calculated for each of the test files:

- 3-D fixed length codes

- 3-D variable length codes

- 2-D variable length codes

Regardless of the coding method used, the file header and track header information remains the same. Table 4 summarises the number of bits used to code these.

Table 4: Bit allocation for headers

\begin{tabular}{|l|c|}
\hline \multicolumn{1}{|c|}{ Aspect } & No of bits \\
\hline $\begin{array}{l}\text { File header } \\
\text { track positions in file }\end{array}$ & $\begin{array}{c}32 \text { bits/track } \\
\text { array terminated by NULL }\end{array}$ \\
\hline Track header & 46 bits/track \\
start time & 16 bits \\
first amplitude & 16 bits \\
first frequency & 14 bits \\
\hline
\end{tabular}

Table 5 summarises the required bits per frame to code the tracks for each coding method. Note each track is terminated by a NULL code of length as indicated.

Table 5: Bit allocation for track contours

\begin{tabular}{|c|c|c|}
\hline Method & Bits per frame & $\begin{array}{c}\text { Terminator length } \\
\text { (bits) }\end{array}$ \\
\hline 3-D fixed & 4.0 & 4 \\
\hline 3-D variable & 3.1 & 5 \\
\hline 2-D variable & 3.5 & 6 \\
\hline
\end{tabular}

The number of tracks and average track length generated using the current implementation of the algorithm is summarised in Table 6 .

Table 6: Number of tracks and average lengths for test files

\begin{tabular}{|c|c|c|}
\hline File name & Number of Tracks & $\begin{array}{c}\text { Average Track } \\
\text { Length }\end{array}$ \\
\hline Telemann & 1614 & 40 frames \\
\hline Bach & 2948 & 32 frames \\
\hline Numbers & 956 & 24 frames \\
\hline Vowels & 1075 & 24 frames \\
\hline
\end{tabular}

summarises the bit rates achieved for each of the test files using the four different methods. The compression ratio is calculated based on the bit rate of the original files, which is $500 \mathrm{kbps}$. 
Table 7: Compression results summary

\begin{tabular}{|c|c|c|c|c|}
\hline \multirow[b]{2}{*}{ File } & \multicolumn{3}{|c|}{ Bit rate (kbps) } & \multirow{2}{*}{$\begin{array}{c}\text { Compression } \\
\text { ratio } \\
\text { (3-D var) }\end{array}$} \\
\hline & 3-D fixed & $\begin{array}{c}\text { 3-D } \\
\text { variable }\end{array}$ & $\begin{array}{c}2-\mathrm{D} \\
\text { variable }\end{array}$ & \\
\hline Telemann & 84.7 & 72.5 & 78.5 & 6 \\
\hline Bach & 134.3 & 116.6 & 125.4 & 4 \\
\hline Numbers & 36.9 & 32.7 & 34.9 & 15 \\
\hline Vowels & 41.5 & 36.7 & 39.2 & 13 \\
\hline
\end{tabular}

\subsection{Discussion of results}

shows that the average compression ratio is approximately $9: 1$. This compares favourably with the $6: 1$ achieved using lossless MPEG layer 3 for $\mathrm{CD}$ quality audio. However, it does not compare favourably with the performance of perceptually lossy MPEG which can achieve compression ratios of as much as $24: 1$. It would be possible to further reduce the bit rate using less structured coding techniques. However, this would come at the expense of obfuscating the revealed data structure in the representation. As was stressed in the introduction, this is counter to the aims of the current work.

In the current implementation, the reconstructed audio signals do display a small degree of pre-echo. This problem will be addressed by initially exploiting temporal information in higher bands to 'fine tune' low band onsets. Further pre-echo cancellation processing will be considered if necessary.

The relative frequency of transitions along tracks reported in Table 3 also bears interest for the interaction and retrieval aspects of the representation. It is apparent from this data that deciding whether a group of tracks represents music or speech can be determined by performing a simple statistical analysis of the tracks. Specifically, musical tracks display relatively few frequency fluctuations (up or down) and have amplitude transitions that are equally distributed among the three possibilities. Speech on the other hand, displays a greater proportion of frequency fluctuations and fewer amplitude fluctuations. With a little additional complexity, the same information can also be extracted from the 3-D tracks. A larger sample of music and speech files will need to be tested to verify this trend. However, the result appears to support the intuition that speech will generally display a concave formant structure while music is composed primarily of horizontal tracks.

\section{Conclusions and future work}

This paper has described a compact, structured audio representation designed specifically for content-based retrieval and interactive data applications. This has been followed by an investigation of the storage requirements of the representation based on an information preserving coding scheme. Finally, the resulting compression gains have been reported for both music and speech signals.

It has been shown that in principle, it is possible to provide compressed audio storage simultaneously with content based retrieval support. Using the representation developed and not compromising it's suitability for retrieval, an expected compression ratio of approximately 6:1 has been calculated. This is due in part to the fact that some of the features inherent to the representation readily support compression. In particular, it's perceptual base results in perceptually redundant data being discarded. Further compression can be achieved by using appropriate coding techniques. However, the aim of maintaining direct access to the perceptually significant attributes and structure of the underlying data remains the paramount concern and thus limits the choice of coding techniques.

While the main aim of this research is to explore aspects of content based retrieval based on a structured audio representation, initial coding issues have been investigated. Further work on compressed representation is left to be performed at a later date. However, given the voluminous nature of audio data it would be unwise to provide retrieval facilities in complete ignorance of compression issues. Indeed, data compression can aid in the retrieval task by reducing the volume of data to be searched. Thus, rather than solely focussing on retrieval aspects, developing a fully coded version of the representation has been necessary to gain an initial indication of the compression that is possible.

\section{References}

[1] K. Melih and R. Gonzalez, "Audio Retrieval Using Perceptually Based Structures", Proc. IEEE International Conf. On Multimedia Computing and Systems '98, Austin, Texas, 28 June - 1 July 1998, 338-347.

[2] N. S. Jayant and E. Y. Chen, "Audio Compression: Technology and Applications", AT\&T Technical Journal, March/April 1995, pp 23-34.

[3] R. Gonzalez, "Hypermedia Data Modelling, Coding and Semiotics", Proc of the IEEE, vol. 85, no. 7, July 1997, pp 1111-1141.

[4] E. Wold, T. Blum, D. Keislar and J. Wheaton, "ContentBased Classification, Search and Retrieval of Audio, IEEE Multimedia, Fall 1996, pp. 27-36.

[5] N. Jayant, J. Johnston and T. Safranek, "Signal Compression Based on Models of Human Perception", Proc of the IEEE, vol. 81, no. 10, Oct 1993, pp1383-1421.

[6] R.N. Bracewell, The Hartley Transform, Oxford University Press, NY, 1986.

[7] P.V.C. Hough, "Method and means for recognising complex patterns", U.S. Patent 3069654 (1962).

[8] R.J. McAulay, T.F. Quatieri, "Speech Analysis/ Synthesis Based on a Sinusoidal Representation", IEEE Trans on ASSP, vol ASSP-34, no 4, August 1986, 744-754.

[9] H. Freeman, "Computer processing of line drawing images", Computing Surveys, vol. 6, March 1974, pp 57-97. 